LAWRENCE LIVERMORE N A T IO N A L LABORATORY
Structural Stability And Optical Properties Of Nanomaterials With Reconstructed Surfaces

A. Puzder, A.J. Williamson, F.A. Reboredo, G. Galli

November 6, 2003

Physical Review Letters 
This document was prepared as an account of work sponsored by an agency of the United States Government. Neither the United States Government nor the University of California nor any of their employees, makes any warranty, express or implied, or assumes any legal liability or responsibility for the accuracy, completeness, or usefulness of any information, apparatus, product, or process disclosed, or represents that its use would not infringe privately owned rights. Reference herein to any specific commercial product, process, or service by trade name, trademark, manufacturer, or otherwise, does not necessarily constitute or imply its endorsement, recommendation, or favoring by the United States Government or the University of California. The views and opinions of authors expressed herein do not necessarily state or reflect those of the United States Government or the University of California, and shall not be used for advertising or product endorsement purposes. 



\title{
Structural stability and optical properties of nanomaterials with reconstructed surfaces
}

\author{
Aaron Puzder, A.J. Williamson, ${ }^{*}$ F.A. Reboredo, and Giulia Galli \\ Lawrence Livermore National Laboratory, Livermore, CA 94550
}

\begin{abstract}
We present density functional and quantum Monte Carlo calculations of the stability and optical properties of semiconductor nanomaterials with reconstructed surfaces. We predict the relative stability of silicon nanostructures with reconstructed and unreconstructed surfaces, and show that surface step geometries unique to highly curved surfaces dramatically reduce the optical gaps and decrease excitonic lifetimes. These predictions provide an explanation of both the variations in the photoluminescence spectra of colloidally synthesized nanoparticles and observed deep gap levels in porous silicon.
\end{abstract}

One of the key features that distinguishes nanomaterials from their bulk counterparts is their large surface to volume ratio. This predominance of the surface is responsible for many of the novel physical properties of nanomaterials. Recently, nanostructure surfaces have been shown to influence properties as varying from surface trap suppression of gain in quantum dot lasers[1] to the resonant frequencies and energy loss in NEMS resonators[2]. Understanding and controlling surfaces is particularly important in semiconductor nanostructures, where reconstructions are expected to play a key role. Current experimental difficulties fabricating and imaging nanostructured materials with curved surfaces place $a b$ initio theoretical calculations as one of the most promising tools for understanding these surfaces.

In this Letter we present a series of ab-initio calculations of nanostructure surfaces, using silicon nanoparticles as a prototype system. These calculations are the first Quantum Monte Carlo (QMC) study of the formation energies of clusters larger than a few atoms, extending the scope of QMC to the study of surface formation energies. We examine a set of atomic surface and step reconstructions, present only on highly curved nanoparticles. The thermodynamic stability of these nano-surfaces are predicted under different synthesis conditions. The effects of these surface reconstructions on the opto-electronic properties are also examined. Our results show that while the optical properties of very small nanoparticles (less than $1 \mathrm{~nm}$ ) are strongly influenced by the presence of surface dimers, the presence of surface steps is actually responsible for the largest optical gap reductions in most nanoparticles. These predictions are applicable to a wide variety of semiconductor nanostructures with highly curved surfaces such as quantum dots and wires, NEMS devices and nano-cantilevers.

Early theoretical studies of nanoclusters simulated the surface by simply enforcing vanishing boundary conditions on the electronic wave functions. Recently, more sophisticated atomistic calculations have been reported using semi-empirical tight binding[3] and pseudopotential[4] approaches. While these methods accurately reproduce the core, bulk-like regions of nan- oclusters, their description of the surface is limited to fitting a surface potential to experimental spectra[4]. These calculations [5] demonstrated that the optical properties strongly depend on this choice of surface potential. To eliminate these ambiguities in the choice of surface potential, first principles calculations of nanocluster surfaces are required. To date, only surface dimers on ultrasmall 29 atom silicon clusters[6] have been studied. The size dependence of the effects of these dimers remains unknown and the relative stability of alternative surface structures is yet to be explored.

To investigate both the stability of different nanostructure surface reconstructions and the importance of the nanocluster surface to volume ratio, we studied spherical silicon clusters ranging in size from 53 to 331 atoms $(0.7$ to $2.0 \mathrm{~nm})$. The clusters were modeled with diamond structure cores and surface dangling bonds were terminated with $\mathrm{SiH}$ and $\mathrm{SiH}_{2}$ groups. To generate nanoclusters with reconstructed surfaces a two step approach was adopted. First, (100)-type facets were reconstructed to a $(2 \times 1)$ configuration by removing a hydrogen atom from neighboring pairs of $\mathrm{SiH}_{2}$ groups, forming an additional Si-Si bond (see inset a in Fig. 1). For the larger clusters ( $\geq 148$ atoms), steps on the (100) facets can also be reconstructed (see inset b in Fig. 1), further reducing the hydrogen coverage of the surface. All atomic structures were relaxed using density functional calculations[7], within the local density approximation (LDA).

Fig. 1 shows the atomic structures of two $1.8 \mathrm{~nm}$ clusters: (i) $\mathrm{Si}_{148} \mathrm{H}_{120}$ which has an unreconstructed surface and (ii) $\mathrm{Si}_{148} \mathrm{H}_{72}$ where neighboring dihydrides $\left(\mathrm{SiH}_{2}\right)$ on the $(100)$ facets have been reconstructed to a $(2 \times 1)$ configuration, similar to the bulk (100) surface reconstruction[8] and the steps between (100) facets have also been reconstructed. The silicon atoms forming one of the reconstructed dihydrides and one of reconstructed steps have been colored yellow and red respectively in $\mathrm{Si}_{148} \mathrm{H}_{72}$. On the surface of this cluster there are 12 of each of these configurations. One might expect the core $\mathrm{Si}-\mathrm{Si}$ bond lengths to be shorter than in bulk silicon due to the "surface tension" produced by reconstruction. 
(i) $\mathrm{Si}_{148} \mathrm{H}_{120}$

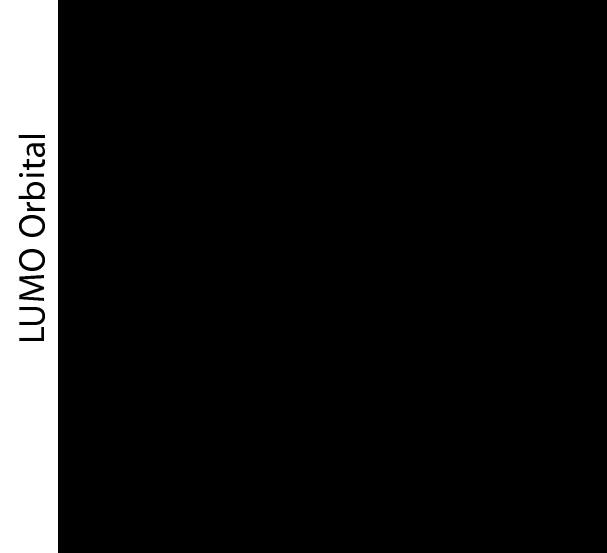

(ii) $\mathrm{Si}_{148} \mathrm{H}_{72}$

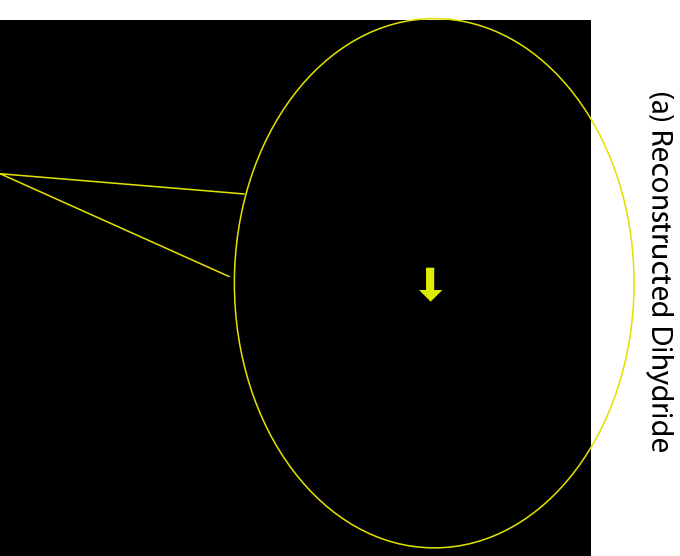

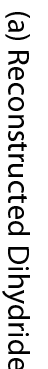

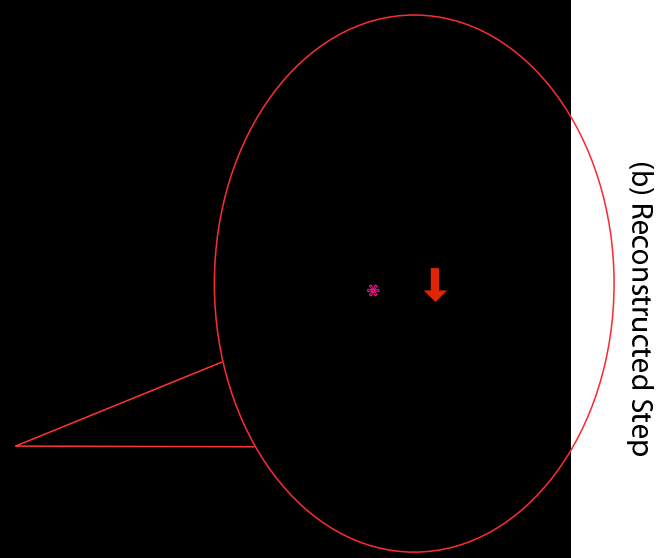

FIG. 1: Unreconstructed $\left(\mathrm{Si}_{148} \mathrm{H}_{120}\right)$ and reconstructed $\left(\mathrm{Si}_{148} \mathrm{H}_{72}\right)$ hydrogen (white atoms) passivated $1.8 \mathrm{~nm}$ silicon (grey atoms) nanoclusters. Charge density isosurfaces (blue) represent $50 \%$ peak amplitude.

However, we find core bond lengths $<1 \%$ shorter than the bulk value in both clusters. The bond lengths of the $(2 \times 1)$ dimers and the reconstructed steps are similar to those predicted by LDA calculations for flat, hydrogen passivated (001) surfaces[9] and reconstructed steps on a (100) surface[10]. After reconstructing a step edge, the silicon atoms with 4 silicon neighbors $(*$ in inset b of Fig. 1) are more constrained producing $\mathrm{Si}-\mathrm{Si}$ bonds that are $\sim 10 \%$ longer than in the core.

In addition to changes in bond lengths we also observe interesting surface structures that are unique to curved nanocluster surfaces. On a flat surface the large number of interacting dihydrides form a "canted" [9] structure to minimize the repulsion between hydrogen atoms. In contrast, the facets on a nanocluster surface are small. For example, the (100) facets of $\mathrm{Si}_{148} \mathrm{H}_{120}$ in Fig. 1(i) have only one pair of dihydrides. These neighboring dihydrides repel each other by rotating in opposite directions (see arrows in Fig. 1), further increasing the $\mathrm{H}-\mathrm{H}$ distance from $1.51 \AA$ to $2.08 \AA$. This additional energetic advantage of the curved surface is reflected in the negative formation energy of the step on (100) surfaces[10].

To predict the relative stabilities of the unrecon- structed and reconstructed clusters we follow the prescription of Ref [9] for the relative formation energy, $\Omega$, of the clusters

$$
\Omega=E^{T o t}+E_{Z P E} n_{H}-n_{H} \mu_{H}
$$

where $E$ is the total energy, $E_{Z P E}$ is the zero-point energy of $\mathrm{Si}-\mathrm{H}$ vibrations, $n_{H}$ is the number of hydrogen atoms, and $\mu_{H}$ is the chemical potential of hydrogen. As the temperatures being considered here are much lower than the Si-H vibrational energy ( $3000 \mathrm{~K})$, only zero point $\mathrm{Si}-\mathrm{H}$ vibrations are included. Additionally, as the number of $\mathrm{Si}$ atoms is the same in each cluster, the $\mathrm{Si}-\mathrm{Si}$ vibrational contributions to the free energy are assumed to be equal and therefore cancel out of the relative formation energies. In the current work, the structures are first relaxed within the LDA. Then QMC calculations are used to more accurately determine the total energy, $E^{T o t}$, of each cluster. In Fig.2 the QMC formation energies of the $\mathrm{Si}_{148} \mathrm{H}_{X}$ clusters are plotted as a function of the hydrogen chemical potential, $\mu_{H}$. As a qualitative guide the upper x-axis shows the temperature dependence of $\mu_{H}$, computed from the standard partition function, within the harmonic approximation, including both rotational 


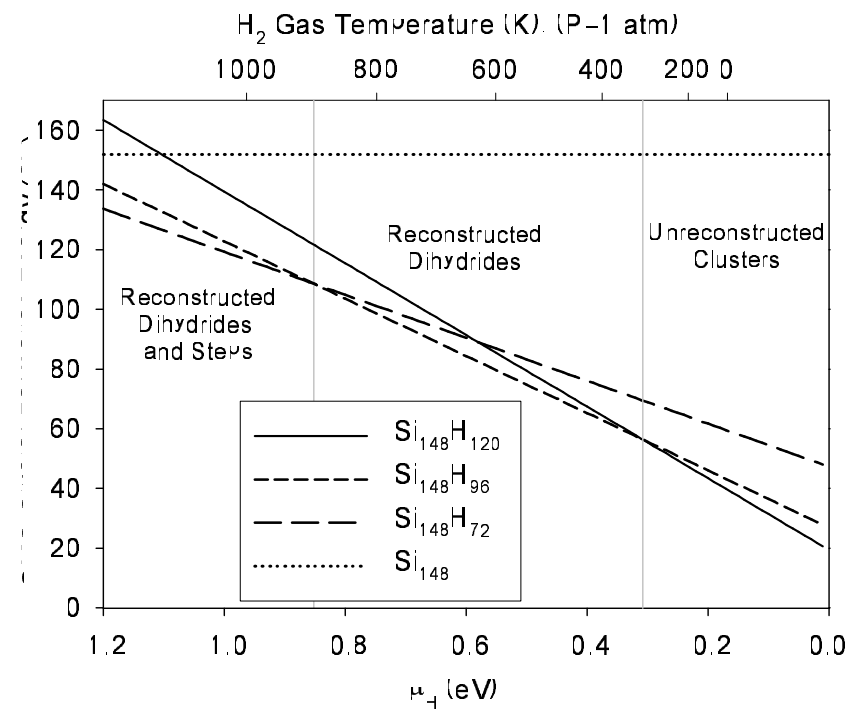

FIG. 2: QMC calculated formation energies of $1.8 \mathrm{~nm} \mathrm{Si}_{148}$ clusters with different surface structures. The origin of $\mu_{H}$ is value at which the formation energy of $\mathrm{SiH}_{4}$ is zero. The value of $\mu_{S i}$ for bulk silicon was obtained from Ref.[11].

and zero-point vibrational energies [19] at 1 atmosphere of partial pressure.

The structure with the highest coverage of hydrogen on the surface, and hence no reconstructions $\left(\mathrm{Si}_{148} \mathrm{H}_{120}\right)$, has the lowest formation energy when $\mu_{H}>-0.3 \mathrm{eV}$. These high $\mu_{H}$ conditions exist when silicon nanoclusters are formed by reducing $\mathrm{SiCl}_{4}$ with metal hydrides[13] or by HF etching of bulk silicon[14]. For nanoclusters synthesized in an $\mathrm{H}_{2}$ environment $\mu_{H}$ is considerably lower. For $\mu_{H}<-0.3$ it is energetically favorable to reconstruct surface dihydrides forming $(2 \times 1)$ surface dimers $\left(\mathrm{Si}_{148} \mathrm{H}_{96}\right)$ and if $\mu_{H}<-0.8 \mathrm{eV}$ reconstructing surface steps to further reduce the hydrogen content $\left(\mathrm{Si}_{148} \mathrm{H}_{72}\right)$ becomes energetically favorable.

The importance of using an accurate many-body approach such as QMC is illustrated by comparing the values of $\mu_{H}$ at which the most stable structure changes from $\mathrm{Si}_{148} \mathrm{H}_{120}$ to $\mathrm{Si}_{148} \mathrm{H}_{96}$ to $\mathrm{Si}_{148} \mathrm{H}_{72}$ to $\mathrm{Si}_{148}$. Our QMC calculations (Fig. 2) predict critical values of $\mu_{H}$ of $-0.3,-0.85$ and $-1.5 \mathrm{eV}$. In contrast, an equivalent LDA calculations predicts crossover at $-0.25,-0.68$ and $-0.85 \mathrm{eV}$. In addition, the QMC phase diagram predicts a positive formation energy for $\mathrm{Si}_{148} \mathrm{H}_{120}$ at $\mu_{H}=0$, i.e. $\mathrm{Si}_{148} \mathrm{H}_{120}$ will not spontaneously form from silane and bulk silicon. LDA calculations predict instead a negative formation energy of $\mathrm{Si}_{148} \mathrm{H}_{120}$ at $\mu_{H}=0$ and hence spontaneous formation of nanoscale clusters.

While LDA calculations have previously been shown to accurately predict structural properties of silicon nanoclusters, they have also been shown[15] to significantly underestimate their optical gaps. Therefore, to determine the effect of surface reconstruction on the optical gap, a series of QMC optical gap calculations were performed for clusters with diameters ranging from 0.7 to $2 \mathrm{~nm}$, using the approach described in Ref.15. For each cluster the lowest energy structure of the unreconstructed and reconstructed surfaces was determined using LDA calculations and the optical gaps were calculated using QMC. The results of these calculations are summarized in Fig. 3a. In Fig.3a we compare QMC calculations of the size dependence of the optical gaps of silicon nanoclusters with unreconstructed surfaces to those with reconstructed dimers and steps on the surface. The optical gap of unreconstructed clusters has previously been shown $[3,4,15]$ to exhibit a strong size dependence, decreasing from $9.1 \mathrm{eV}$ for the smallest limiting case of the $\mathrm{SiH} 4$ silane molecule, to $1.1 \mathrm{eV}$ for clusters approaching the bulk limit. In contrast, Fig.3a shows that clusters with reconstructed surfaces exhibit a much weaker size dependence, most likely due to the surface localized nature of the band edge states. In contrast to the quantum confined core states of unreconstructed clusters, these states behave more like impurity levels that are independent of the size of the cluster. Clusters with only reconstructed dimers have an optical gap of $\sim 3.5 \mathrm{eV}$. Larger clusters ( $\geq 148$ Si atoms) are also able to reconstruct steps on the (001) surface. This double reconstruction dramatically lowers the optical gap to $\sim 1.5 \mathrm{eV}$. Using the trends of the LDA gaps we extrapolate the QMC gaps to larger sizes (dashed line in Fig. 3a) and predict that beyond $2 \mathrm{~nm}$ the unreconstructed optical gap will cross below the gap produced by dihydride to dimer reconstructions. Therefore, the effect of dimer reconstructions will not be observed in clusters $>2 \mathrm{~nm}$. However, as reconstructing surface steps lowers the optical gap energy to only slightly above the bulk band gap, we predict it should be possible to observe the optical signature of step reconstructions in clusters as large as 5-10nm.

The nature of a nanocluster surface can also be characterized by the excitonic lifetime. Fig.3b shows the size dependence of the exciton lifetime in clusters with unreconstructed surfaces and surfaces with reconstructed dihydrides calculated within LDA using the procedure in Ref.[16]. For clusters with diameters $>1.3 \mathrm{~nm}$ the exciton created by a HOMO to LUMO excitation (solid circles and squares) is plotted. In the smallest clusters, excitations between band edge states slightly below(above) the HOMO(LUMO) (empty symbols) with the same surface character as the HOMO and LUMO in the larger clusters are plotted as these have significantly shorter lifetimes. For unreconstructed clusters, the exponential increase in lifetime of the exciton (circles connected by solid line in Fig.3b) is consistent with previous calculations[17] and is interpreted as the HOMO to LUMO transition becoming more "indirect" as the bulk limit is approached. For clusters with reconstructed dihydrides, the lived exciton exhibits a similar exponential size dependence, but the lifetime is approximately an order of magnitude shorter 


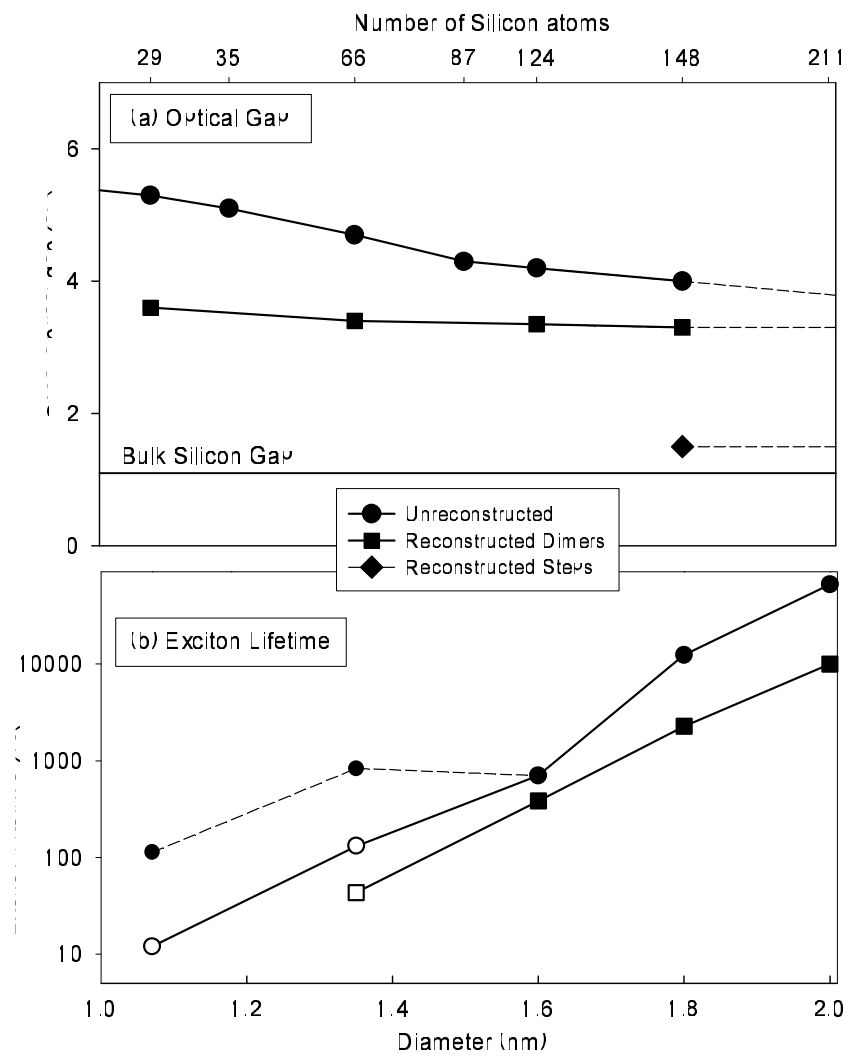

FIG. 3: (a) Optical gaps of nanoclusters with different surface structures. Dashed lines are extrapolations based on LDA trends. (b) Solid circles(squares) show LDA exciton lifetimes of unreconstructed (reconstructed) clusters. Empty symbols show the lifetime of states with $\mathrm{HOMO}(\mathrm{LUMO})$ character just below(above) the band edge.

for all sizes.

In Ref.[18], van Buuren et. al use X-ray absorption and valence band photoemission to investigate the shift in the valence and conduction band edges of porous silicon as a function of thermal annealing. The wavelength of the observed photoluminescence suggests that the optical emission in these samples originates from nanoscale features with dimensions of $\sim 2 \mathrm{~nm}$, similar to the nanoclusters studied here. As the annealing temperature is increased, mass spectrometer measurements showed significant hydrogen evolution coupled with a decrease in the quantum confinement of the conduction and valence bands. At an annealing temperature of $600 \mathrm{~K}$ the quantum confinement of the conduction and valence bands is reduced by 0.6 and $0.4 \mathrm{eV}$ respectively, effectively reducing the optical gap by $1 \mathrm{eV}$. This evolution of hydrogen at $600 \mathrm{~K}$ is consistent with the reconstruction of surface steps predicted in Fig. 2 and the measured $1 \mathrm{eV}$ gap reduction is close to the $1.5 \mathrm{eV}$ reduction due to surface reconstruction predicted in Fig. 3a.

Evidence for the existence of silicon nanoclusters with reconstructed surfaces is also provided in Ref.[13] in which absorption and emission spectra of $1.8 \mathrm{~nm}$ clusters synthesized in inverse micelles are presented. Our QMC calculations of the optical gaps of clusters with reconstructed surface dimers and steps of exactly this size (3.5 and $1.5 \mathrm{eV}$, see Fig. 3) are in good agreement with the two gaps of 3.4 and $2.1 \mathrm{eV}$ measured in Ref.[13]. Our calculations therefore strongly support the conjecture of Ref.[13] that the smaller of these two optical gaps (2.1 $\mathrm{eV}$ ) is due to surface recombination.

In conclusion, we have report the first ab-initio investigation of the relative stability of silicon nanoparticles with reconstructed surfaces as a function of size and we relate our results to specific experimental preparation conditions. Our findings show that the relative stability of silicon nanoparticles as a function of hydrogen content and surface structure is very different from that extracted from known results for flat surfaces. In addition, we have describe, in detail, surface step reconstructions of hydrogenated silicon nanoparticles, which we found to occur in dots larger than 150 atoms, and which we suggest are responsible for the dramatic shifts in optical gaps observed experimentally in porous silicon and quantum dots after annealing. The mechanism identified here as responsible for the optical gap reduction generates gap shifts seven times larger than the surface dimer bond model previously considered for ultrasmall nanoparticles and it thus explains several different experimental results as a function of size. Finally, we discuss the size dependence of exciton lifetimes (and hence the experimental quantum efficiency) of silicon nanoclusters and propose a description of the optical and structural properties of silicon nanoparticles which is consistent with X-ray and photoluminescence studies of both colloidally synthesized samples and porous silicon.

We thank Richard Needs for providing the QMC calculated value of $\mu_{S i}$ used in calculating Fig. 2. We also thank Randolph Hood and Jeffrey Grossman for performing QMC calculations. This work was performed under the auspices of the U.S. Department of Energy by the University of California, Lawrence Livermore National Laboratory under contract No. W-7405-Eng-48.

* Electronic address: williamson1001lnl.gov

[1] V. Klimov, A. Mikhailovsky, S. Xu, Malko.A., J. Hollingsworth, C. Leatherdale, H. Eisler, and M. Bawendi, Science 290, 314 (2000).

[2] M. Roukes, Sci. Amer. 48, 285 (2001).

[3] C. Delerue, G. Allan, and M. Lannoo, Phys. Rev. B 48, 11024 (1993).

[4] L. Wang and A. Zunger, J. Phys. Chem. 98, 2158 (1994).

[5] F.A. Reboredo and A. Zunger, Phys. Rev. B 63, 235314 (2001).

[6] G. Allan et al., Phys. Rev. Lett. 76, 2961 (1996); L. Mitas et al., Appl. Phys. Lett. 78, 1918 (2001); I. Vasiliev and R. Martin, Phys. Stat. Solidi B 233, 5 (2002). 
[7] The GP Code (F. Gygi, LLNL 2002), with a plane wave basis (35 Ry cutoff) and norm-conserving pseudopotentials was used for all DFT calculations. The LDA was used throughout. Representative clusters were compared with the gradient corrected PBE functional, which produced negligible changes in the structural and optical properties.

[8] G.P. Kerker, S.G. Louie, and M.L. Cohen, Phys. Rev. B 17, 706 (1978).

[9] J.E. Northrup, Phys. Rev. B 44, 1419 (1994).

[10] F.A. Reboredo, S.B. Zhang, and A. Zunger, Phys. Rev. B 63, 125316 (2001).

[11] P.R.C. Kent, R.Q. Hood, A.J. Williamson, R.J. Needs, W.M.C. Foulkes, and G. Rajagopal, Phys. Rev. B 59, 1917 (1999).

[12] All QMC calculations were performed using the CASINO QMC code, version 1.5.4 User's Manual R. Needs et. al, University of Cambridge (2002).

[13] J.P. Wilcoxon, G.A. Samara, and P.N. Provencio, Phys.
Rev. B 60, 2704 (1999).

[14] G. Belomoin, J. Therrein, and M. Nayfeh, Appl. Phys. Lett. 77, 779 (2000).

[15] A.J. Williamson et al., Phys. Rev. Lett. 89, 196803 (2002) and references therein.

[16] A. Puzder, A. Williamson, J. Grossman, and G. Galli, J. Chem. Phys 117, 6721 (2002).

[17] B. Delley and E.F. Steigmeier, Phys. Rev. B 47, 1397 (1993).

[18] T. vanBuuren, T. Tiedje, S.N. Patitsas, and W. Weydanz, Phys. Rev. B 50, 2719 (1994).

[19] $\mu_{H}(T)$ was calculated[20] by $\mu_{H_{2}}=E_{H_{2}}+\hbar \omega_{H_{2}} / 2+$ $k_{B} T \ln \left[n \Lambda^{3} \theta_{r} / T\right]$, where $\hbar \omega_{H_{2}} / 2$ is the zero point vibrational energy, $n$ is the density, $\theta_{r}=85.4 K$ and $\Lambda=\frac{2 \pi \hbar}{\sqrt{4 m \pi K T}}$

[20] G. S. Rushrooke, "Introduction to Statistical Mechanics", Oxford (1957). 